\title{
Comparison of the Flagellins from Different Flagellar Morphotypes of Escherichia coli
}

\author{
By A. M. LAW N* \\ Lister Institute of Preventive Medicine, London SWI W $8 R H$ \\ (Received 9 February 1977)
}

\begin{abstract}
The molecular weights of the flagellins of I 3 strains of Escherichia coli, each with a different $\mathrm{H}$ antigen, were estimated using polyacrylamide gel electrophoresis. In each case only one major polypeptide was demonstrated, although some strains possessed apparently sheathed flagella. Considerable differences in the molecular weight of flagellin accompanied the previously described structural differences between flagella from strains with different $\mathrm{H}$ antigens. The relationship between flagellar diameter and the molecular weight of the corresponding flagellins was similar for both unsheathed and apparently sheathed flagella. Cross-polymerization occurred between seed consisting of fragments of unsheathed flagella and flagellin solution from apparently sheathed flagella and vice versa. Co-polymerization of flagellin from unsheathed flagella and flagellin from apparently sheathed flagella was also demonstrated. These polymerization experiments indicate that the assembly pattern of flagellin molecules is probably the same in all $E$. coli flagella. The above and other evidence suggests that there is no true sheath, but that the differences in flagellar surface structure between different $E$. coli flagella are the result of differences in the superficial parts of the flagellin molecules.
\end{abstract}

\section{INTRODUCTION}

Considerable variation of surface structure exists between flagella from different $\mathrm{H}$ serotypes of $E$. coli, which can be grouped into different morphotypes (Lawn, Ørskov \& Ørskov, I977). The flagellar filaments of some morphotypes have a surface structure which, in other genera of enterobacteria, has been attributed to the presence of a separate sheath (Lowy \& Hanson, 1965; Schmitt, Raska \& Mayer, 1974). This account describes investigations designed to detect the presence of a separate sheath protein or differences in the pattern of assembly of the protein units to form the filaments of different flagellar morphotypes. The use of sodium dodecyl sulphate-polyacrylamide gel electrophoresis was expected to reveal the presence of an additional sheath protein, and major differences in the pattern of assembly of flagellin, the protein subunit of flagella, were expected to preclude cross-polymerization (block co-polymerization; Asakura, Eguchi \& Iino, 1966).

\section{METHODS}

Bacterial strains. The strains of $E$. coli were all from the collection of standard $H$ reference strains at the Collaborative Centre for Reference and Research on Escherichia coli (WHO), Copenhagen. Before they were used for preparing purified flagella they were passaged through Craigie tubes containing motility medium with a gelatine and agar basis. Such highly motile variants could be stored in liquid nitrogen without loss of motility.

* Present address: Houghton Poultry Research Station, Houghton, Huntingdon, Cambridgeshire PEI7 2DA. 
Preparation of purified flagella. A 51 flask containing $x 1$ Oxoid nutrient broth no. 2 was inoculated with a culture of a highly motile variant of the required strain and grown with shaking at $37^{\circ} \mathrm{C}$ for 8 to $16 \mathrm{~h}$. The bacteria with attached flagella were harvested by low-speed centrifugation, resuspended in a small amount of phosphate-buffered saline (PBS, Dulbecco's solution A, $\mathrm{pH} 7.4$ ) and treated in a Waring-type blender to release the flagella. The bacteria were removed by centrifugation and washed with a small volume of PBS. The washings and the original supernatant were combined, and the flagella were recovered by high-speed centrifugation and resuspended in distilled water. The preparations were purified by alternate high and low speed centrifugation in distilled water until the pellet of flagella appeared almost homogeneous. The flagella from $\mathrm{I} 1$ of culture suspended in I $\mathrm{ml}$ distilled water formed the stock suspensions for use in all the experiments; their concentration varied considerably between batches. Because of the small scale of the preparations, protein estimation and adjustment of protein concentration was not attempted.

Sodium dodecyl sulphate-polyacrylamide gel electrophoresis. The discontinuous method described by Maizel (1971) was followed. The samples were heated in a boiling water bath for $90 \mathrm{~s}$ in the presence of sodium dodecyl sulphate (SDS) and mercaptoethanol and cooled before layering under the electrode buffer covering the upper stacking gel of tubes containing approximately I cm of stacking gel $[2 \%(\mathrm{w} / \mathrm{v})$ acrylamide] and approximately $12 \mathrm{~cm}$ of separating gel $[8$ or $10 \%(\mathrm{w} / \mathrm{v})$ acrylamide]. The electrode, sample and stacking gel buffers $(\mathrm{pH} 9)$, the separating gel buffer $(\mathrm{pH} 7)$ and the marker dye were as described by Maizel (197I). The tubes were run at $3 \mathrm{~mA}$ per tube until the sharp marker dye band was a few millimetres from the lower end of the tube. Protein bands were stained with Coomassie brilliant blue.

Determination of molecular weight. Four reference proteins were used: phosphorylase from rabbit muscle (mol. wt 93000), bovine serum albumin (65000), glyceraldehyde phosphate dehydrogenase $(36000)$ and carbonate dehydrogenase ( 31000$)$. In each run, one or two tubes were loaded with a mixture of $2 \mu \mathrm{g}$ of each of the standard proteins while other tubes were loaded with both the flagellar protein and two or more of the standards. Migration distances were corrected using the distance travelled by the marker dye in individual tubes and the average distance for each standard was plotted against the logarithm of the molecular weight. The resulting points were joined by a hand-fitted, smooth curve. From this line, which was not exactly linear, the molecular weight corresponding to the average migration distance of the flagellar polypeptide was determined. In preliminary runs large amounts of flagellar protein were loaded (up to O.I ml) in order to estimate the number of distinguishable polypeptides present in each preparation and their concentration. The reproducibility of the method was checked for Hi2 flagellin using the results for nine tubes from three runs and for $\mathrm{H}_{4} 6$ using eleven tubes from five runs. The standard deviations were \pm 2450 (mol. wt $6 \mathrm{r} 920$ ) and \pm 2020 (mol. wt 56350 ) respectively.

Preparation of flagellar protein. The stock suspensions of flagella, or sonicated stock suspensions, were solubilized by treatment with acid or heat. For the former, $\mathrm{O} \cdot \mathrm{I} \mathrm{M}-\mathrm{HCl}$ was added to the stock suspension, or more usually to a $\mathrm{I}: \mathrm{IO}$ dilution of this in distilled water, until the $\mathrm{pH}$ was approximately $3 \cdot 0$. Insoluble material was removed by high-speed centrifugation. The supernatant was then dialysed against distilled water (for gel electrophoresis) or PBS (for polymerization). For heat dissociation, the stock suspension, or sonicated suspension, was heated in a water bath at $65^{\circ} \mathrm{C}$ for the minimum time required to depolymerize the majority of the flagella, as determined by electron microscopy, and then rapidly cooled. The times used were between 5 and 15 min. The resulting solution was cleared of flagellar fragments by high-speed centrifugation.

Polymerization of flagella. The methods for preparing seed and for repolymerizing flagella on to seed followed those used by Asakura \& Eguchi (1964), except that the experiments were carried out with PBS as the vehicle, and a micromethod was used. The seed was obtained by sonication of the stock suspensions of flagella using a miniature ultrasonic bath (Millipore) for three periods of $5 \mathrm{~min}$ each at $5 \mathrm{~min}$ intervals, the tube containing the suspension of flagella being immersed in ice-cold water throughout. The fragments were recovered by high-speed centrifugation and resuspended in PBS to the initial volume. One-tenth of this suspension was diluted $\mathrm{I}$ : ro with PBS to form the seed suspension and the remainder was used to make soluble flagellar protein (flagellin solution) as described above. Equal quantities of seed suspension and flagellin solution ( $25 \mu \mathrm{l}$ of each) were mixed in plastic tubes and left at room temperature for $24 \mathrm{~h}$ when an equal quantity $(50 \mu \mathrm{l})$ of $2.5 \%(\mathrm{w} / \mathrm{v})$ glutaraldehyde in PBS was added. After a further $24 \mathrm{~h}$ fixation the preparations were prepared for electron microscopy. A copper grid, covered with a carbon-coated formvar membrane and subjected to ionic bombardment to render the membrane hydrophilic, was floated face down on $5 \mu$ l of the seed/monomer mixture for several minutes. The grid was then washed in distilled water, uranyl acetate [approximately $0.5 \%(\mathrm{w} / \mathrm{v})$ ] $\mathrm{k}$ as added and the surplus was immediately and rapidly withdrawn. 


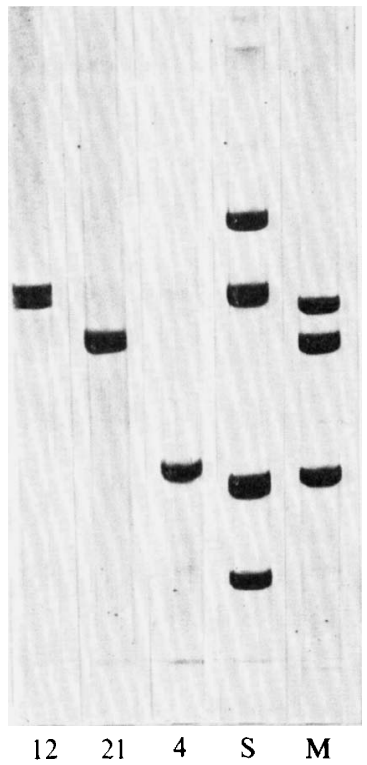

Fig. I. SDS-polyacrylamide gel electrophoresis of flagellins from flagella of serotypes $\mathrm{H}_{2}$, $\mathrm{H}_{2}$ I and $\mathrm{H}_{4}$. The marked difference in mobility between flagellins from the three types of flagella is demonstrated. The gel labelled $M$ was loaded with a mixture of the three proteins, and that labelled S was loaded with four standard proteins: phosphorylase from rabbit muscle (mol. wt 93000), bovine serum albumin (65000), glyceraldehyde phosphate dehydrogenase from rabbit muscle (36000) and carbonate dehydrogenase from bovine erythrocytes $(31000)$.

\section{Table I. Some properties of the flagella and of the corresponding flagellins of different $H$ serotypes of Escherichia coli}

The flagellar morphotypes are described by Lawn et al. (1977). The diameters include the full extent of any regular projections from the flagellar filament and were determined by negative contrast with uranyl acetate of aldehyde-fixed flagella; each value is the mean of ro measurements. Molecular weights were calculated, as described in the Methods, from SDS-polyacrylamide gel electrophoresis of flagellins and standard reference proteins; each value is the mean of a number of estimations (given in parentheses) from individual gels.

\begin{tabular}{|c|c|c|c|c|c|}
\hline Strain & $\begin{array}{l}\text { Flagellar } \\
\text { morpho- } \\
\text { type }\end{array}$ & $\begin{array}{c}\text { H } \\
\text { serotype }\end{array}$ & Surface structure of flagella & $\begin{array}{c}\text { Approx. } \\
\text { diam. of } \\
\text { flagella }(\mathrm{nm})\end{array}$ & $\begin{array}{l}\text { Mol. wt of } \\
\text { flagellin }\end{array}$ \\
\hline U9-4I & $\mathbf{A}$ & 4 & $\begin{array}{l}\text { Thin, long pitch helix } \\
\text { pattern }\end{array}$ & 19 & $37000(2)$ \\
\hline PI $2 b$ & A & I7 & $\begin{array}{l}\text { Thin, long pitch helix } \\
\text { pattern }\end{array}$ & 20 & $39000(2)$ \\
\hline U-4I & B & 5 & Thin, subunit pattern & 20 & $46000(3)$ \\
\hline N234 & B & 25 & Thin, subunit pattern & 20 & $50000(3)$ \\
\hline su 432 I-4I & D & I I & Polar subunit pattern & $2 \mathrm{I}$ & $54000(\mathrm{I})$ \\
\hline UI Ia-44 & D & 2 I & Polar subunit pattern & 22 & $56000(3)$ \\
\hline suI 242 & $\mathrm{E}$ & $\mathbf{I}$ & Short pitch loop pattern & 24 & $60000(6)$ \\
\hline U5-4I & $\mathbf{E}$ & 7 & Short pitch loop pattern & 23 & $61000(6)$ \\
\hline ві3 I 6-42 & $\mathrm{E}$ & 12 & Short pitch loop pattern & 22 & $62000(9)$ \\
\hline $78 \mathrm{I}-55$ & $\mathrm{~F}$ & 44 & Long pitch loop pattern & 25 & $59000(3)$ \\
\hline $5306-56$ & $\mathbf{F}$ & 46 & Long pitch loop pattern & 24 & $56000(\mathrm{I} 1)$ \\
\hline Bi7575-4I & - & 9 & $\begin{array}{l}\text { Thick, long pitch loop } \\
\text { pattern }\end{array}$ & 27 & $69000(3)$ \\
\hline $5017-53$ & - & 36 & Thick, rope-like pattern & 25 & $61000(6)$ \\
\hline
\end{tabular}




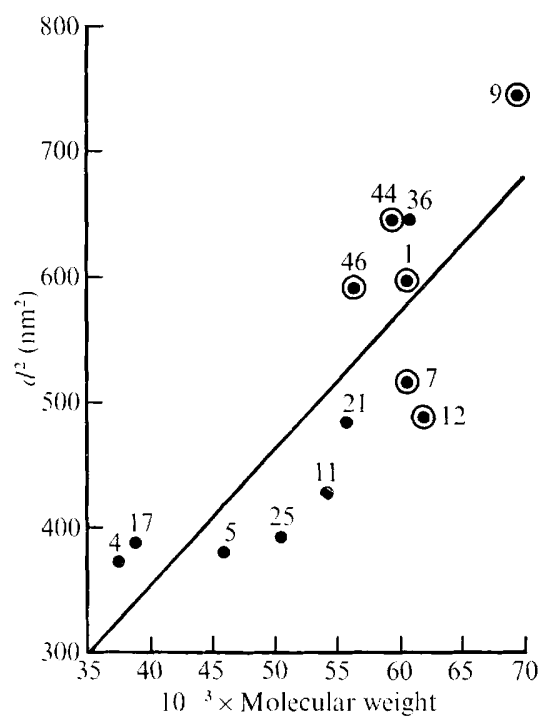

Fig. 2. The relationship between the diameter of the flagellar filament and the molecular weight of the corresponding flagellin for different $\mathrm{H}$ serotypes of Escherichia coli. There is no consistent difference in distribution between the ringed $\boldsymbol{O}$, which represent flagella whose surface structure suggests the presence of a sheath, and the remaining $\boldsymbol{O}$, which represent conventional flagella. The straight line is the regression line of $d^{2}$ on molecular weight.

\section{RESULTS}

Number of polypeptides present in solubilized protein from $E$. coli flagella of different morphotypes

When acid-soluble and heat-soluble protein from $E$. coli flagella of 13 different serotypes was examined by discontinuous SDS-polyacrylamide gel electrophoresis (Fig. I), in every case only one major band was consistently observed. The flagella were prepared from the standard reference strains of $\mathrm{H}$ serotypes I, 4, 5, 7, 9, II, I 2, I7, 2I, 25, 36, 44 and 46, and included examples of five of the six principal morphotypes described by Lawn et al. (I977) (Table I). In a few preparations a flagellin band was split, but this could be attributed to technical inadequacies and did not occur consistently for any one serotype. Faint additional bands were sometimes observed. The single major band was taken to be the polypeptide, flagellin, from which the flagellar filament is assembled (see Smith \& Koffler, I97I).

\section{Molecular weight of flagellins of various serotypes of E. coli}

The molecular weights of the flagellins of 13 serotypes (Table 1 ) were determined by SDS-polyacrylamide gel electrophoresis using standard reference proteins. A considerable range of molecular weights was observed, from 37000 for $\mathrm{H}_{4}$ flagellin to 69000 for $\mathrm{H}_{9}$ flagellin.

\section{Relationship between the molecular weight of flagellin and the diameter of the corresponding flagellum}

When the molecular weights of flagellins from different $E$. coli flagella were plotted against the square of the diameter $(d)$ of the corresponding flagellum there was apparently no consistant difference in the ratio of $d^{2} / \mathrm{mol}$. wt between flagellin from morphotypes which have sheath-like surfaces and those having conventional surfaces (Fig. 2). This result is compatible with a model in which variation in diameter of $E$. coli flagella between different serotypes is due simply to variations in the size of the polypeptide chain of flagellin, without variation in the pattern of assembly of the polypeptides in the flagellar filament. 
Table 2. Cross-polymerization of flagella seed fragments and flagellin solutions from different serotypes of Escherichia coli

The rows represent seed (fragments of flagella produced by sonication) of different types. The flagellar morphotypes are listed in Table $\mathrm{I}$. The columns represent solutions of flagellin obtained by heat $(\mathrm{H})$ or acid (A) treatment of suspensions of flagella from different serotypes (see Methods).

Flagellin

\begin{tabular}{|c|c|c|c|c|c|c|c|}
\hline \multicolumn{2}{|c|}{ Serotype } & $\mathrm{H}_{5}(\mathrm{H})$ & $\operatorname{HI}(\mathrm{A})$ & HI I $(H)$ & $\mathrm{H} 2 \mathrm{I}(\mathrm{H})$ & $\mathrm{H}_{7}(\mathrm{H})$ & $\mathrm{H}_{4} 6(\mathrm{~A})$ \\
\hline \multirow{9}{*}{ Seed } & (H4 & NT & NT & + & NT & NT & + \\
\hline & $\mathrm{Hi} 7$ & - & + & NT & NT & + & + \\
\hline & $\mathrm{H}_{5}$ & + & - & $\mathrm{NT}$ & $\mathrm{NT}$ & + & + \\
\hline & HII & - & NT & $\mathrm{NT}$ & + & NT & - \\
\hline & $\mathrm{H}_{2} \mathrm{I}$ & - & $\mathrm{NT}$ & + & $\mathrm{NT}$ & - & - \\
\hline & $\mathrm{HI}$ & - & - & + & $\mathrm{NT}$ & $\mathrm{NT}$ & + \\
\hline & $\mathrm{H}_{44}$ & - & - & + & $\mathrm{NT}$ & NT & NT \\
\hline & $\mathrm{H}_{4} 6$ & + & + & + & NT & - & NT \\
\hline & $\mathrm{H}_{36}$ & + & - & + & NT & $(+)$ & $(+)$ \\
\hline
\end{tabular}

+ , Polymerization of flagellar filaments from the flagellin defined by the corresponding column occurred on the seed defined by the intersecting row; $(+)$, possible but doubtful polymerization; - , absence of detectable polymerization using the method described in text; NT, not tested.

\section{Cross-polymerization (block co-polymerization) of flagellin from one flagellar morphotype on the seed of another}

Seed was prepared from the flagella of nine different serotypes and solubilized flagellin from eight different serotypes. Only flagellin preparations which gave polymerization with one or more seed preparations are included in Table 2. The flagellin was prepared either by heating or by acid treatment of the sonicated fragments of flagella [used as seed, as described in Methods.

In the first experiment seed of $\mathrm{HI}_{\mathrm{I}}$ and $\mathrm{H}_{2} \mathrm{I}$ were used with the opposite monomers. As these serotypes both have flagella of morphotype D (polar subunit pattern, Lawn et al., I977), the seed and newly polymerized filament needed to be distinguished by using specific antiserum (Asakura et al., 1966) derived by cross-absorbing antiserum to Hi I flagella with $\mathrm{H}_{2}$ I flagella. Only short lengths of $\mathrm{H}_{2}$ I polymer were polymerized on $\mathrm{HI}_{\text {I }}$ seed but $\mathrm{HI}_{\mathrm{I}}$ flagellin gave up to several wavelengths of new polymer on $\mathrm{H}_{2} \mathrm{I}$ seed.

Seed of one morphotype was then mixed with flagellin from a different morphotype in a wide variety of combinations (Table 2). The variation in efficiency of polymerization noted with $\mathrm{HII} / \mathrm{H}_{2} \mathrm{I}$ cross-polymerization was even more marked. Some flagellins, for example heat-dissociated $\mathrm{H}_{9}$, would not polymerize on any seed, although examination of this solution by SDS-polyacrylamide gel electrophoresis indicated that it contained as much polypeptide as others that polymerized efficiently. Other preparations, for example $\mathrm{H}_{4} 6$ and $\mathrm{H}_{7}$, gave only short segments of new polymer whereas heat-dissociated preparations of $\mathrm{H}_{5}$ and $\mathrm{H}_{\mathrm{I}}$ f flagella gave particularly good polymerization.

Heating appears to have a more drastic effect on flagella of some serotypes than on others. A preparation of $\mathrm{Hi} 7$ flagella precipitated after heating at $65^{\circ} \mathrm{C}$ for $\mathrm{I} 5 \mathrm{~min}$ but the supernatant after high-speed centrifugation contained no flagellin detectable by gel electrophoresis. Heating at the same temperature for I min considerably reduced the number of flagella and gave no immediate precipitate but again there was no detectable protein in the supernatant after high-speed centrifugation. The clear supernatant obtained after acid dissociation of $\mathrm{H}_{I 7}$ flagella gave a precipitate when dialysed against $\mathrm{PBS}$ at $\mathrm{pH} 7 \cdot 4$. Examination of this precipitate in the electron microscope revealed no flagella. None of these 'flagellin' preparations gave polymerization of Hi7 filaments with a number of different seed preparations. That the $\mathrm{H}_{9}$ flagellin solution contained defective flagellin is suggested 


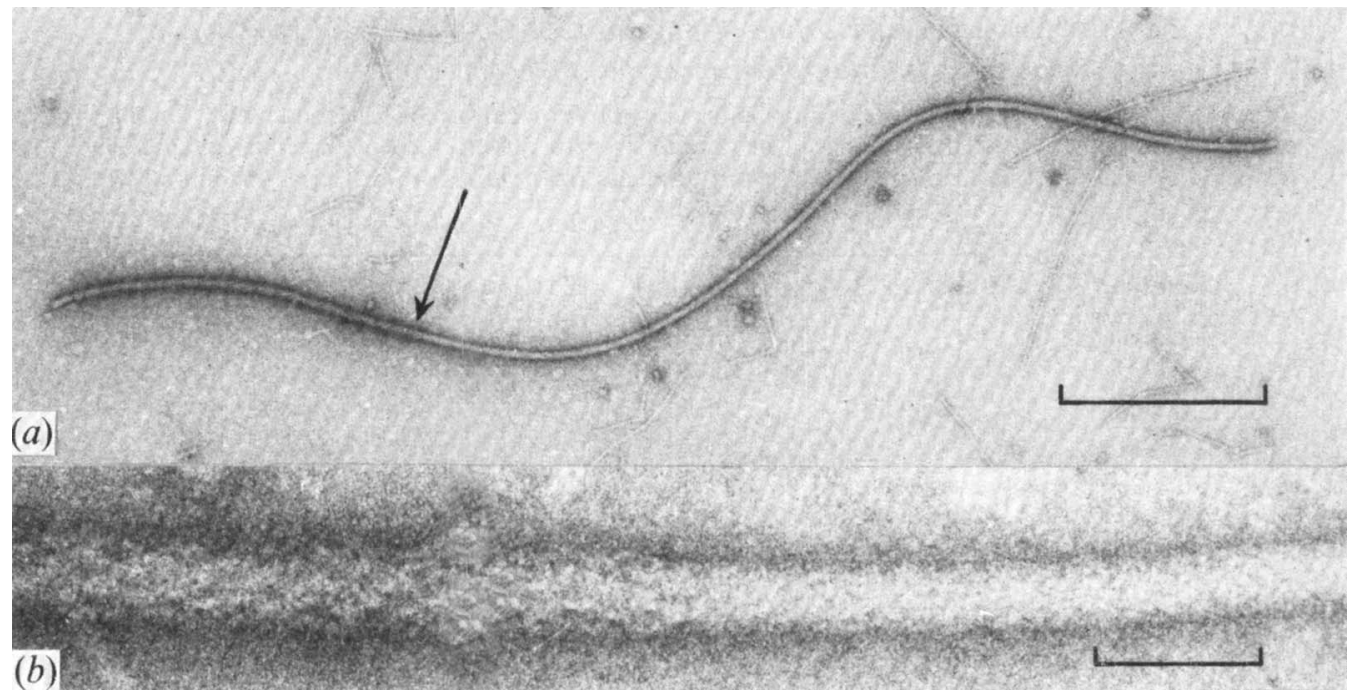

Fig. 3. Cross-polymerization of seed fragments of $\mathrm{H}_{3} 6$ flagella and flagellin solution from $\mathrm{H}_{5}$ flagella. (a) Low-power micrograph showing that the change from seed to new polymer (arrowed) does not interrupt the overall sinusoidal course of the flagellum. Bar marker represents $0.5 \mu \mathrm{m}$. (b) The junction between seed and new polymer at higher magnification. Bar marker represents $50 \mathrm{~nm}$.
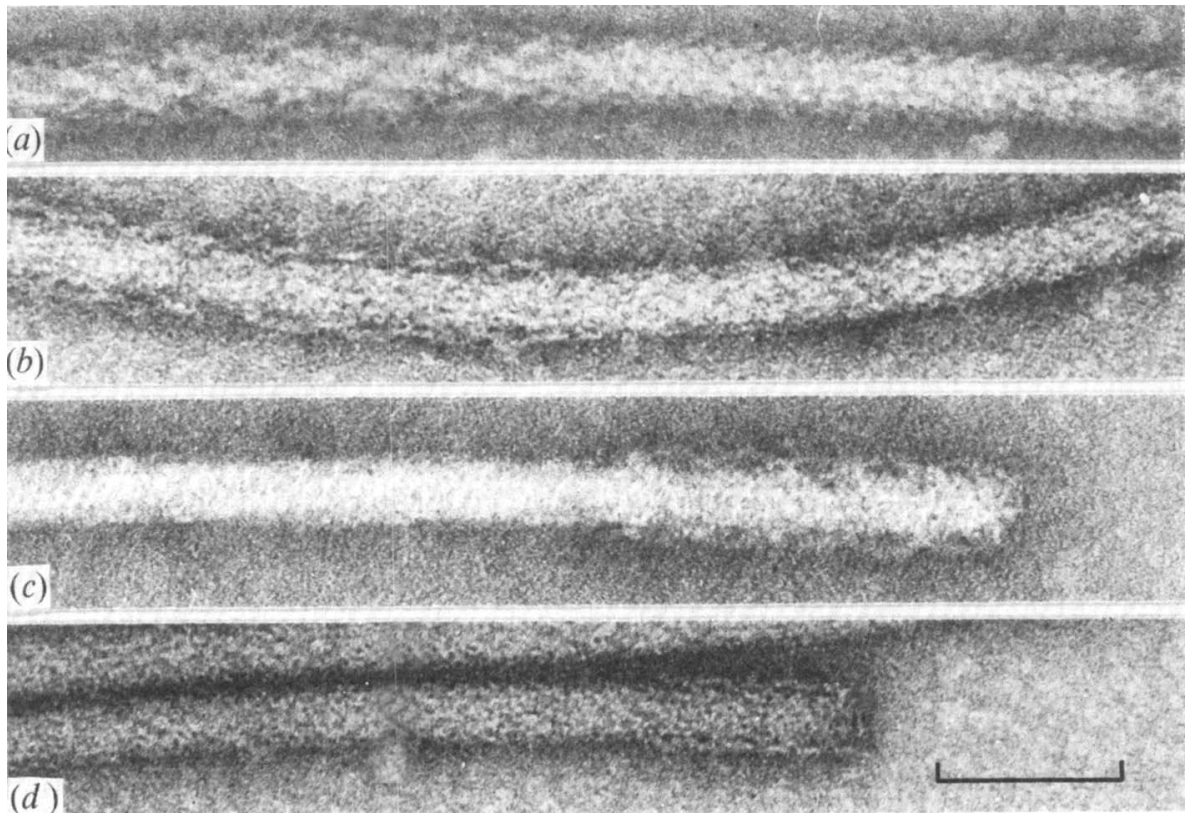

Fig. 4. Cross-polymerization of seed fragments of one flagellar serotype and flagellin solution from another serotype of Escherichia coli. Various combinations are shown, in some of which the seed and the new polymer have entirely different surface structures and diameters, yet join smoothly. The serotypes used were: (a) $\mathrm{H}_{3} 6$ seed and $\mathrm{H}$ I I flagellin; (b) $\mathrm{H}_{4} 6$ seed and HI flagellin; (c) $\mathrm{HI}_{7}$ seed and $\mathrm{H}_{4} 6$ flagellin; $(d) \mathrm{H}_{5}$ seed and $\mathrm{H}_{7}$ flagellin. Bar marker represents $50 \mathrm{~nm}$. 


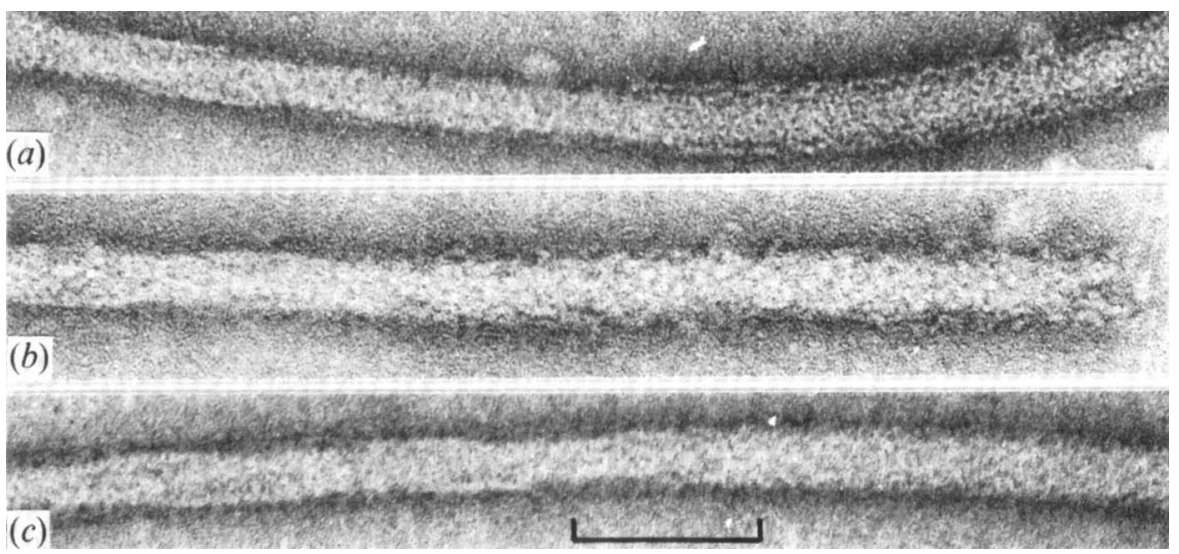

Fig. 5. Co-polymerization between flagellins from flagella of two serotypes on to seed fragments of a third type. $(a),(c)$ Pure flagellin solutions from flagella of $\mathrm{H}_{7}$ and $\mathrm{H}_{5}$ respectively have polymerized on to seed of $\mathrm{H}_{4}$ flagella to give a new filament with a surface pattern characteristic of the parent (right side) and different from that of the seed (left side). (b) A mixture of equal parts of flagellin solutions from $\mathrm{H}_{5}$ and $\mathrm{H}_{7}$ flagella was added to the same seed. A flagellar filament has polymerized which is not characteristic of either $\mathrm{H}_{5}$ or $\mathrm{H}_{7}$ flagella, but is a co-polymer of the two flagellins. Bar marker represents $50 \mathrm{~nm}$.

by results reported in the following section. In view of the results described above no significance should be attached to the negative findings in Table 2. No attempt was made to optimize the conditions for depolymerization or repolymerization of the flagella, neither were repeated attempts made to obtain polymerization, and the concentrations of the stock suspensions of flagella were not equal.

In spite of these limitations cross-polymerization was obtained with a number of different combinations some of which gave new filaments attached to seed of an entirely different morphotype (Figs 3 and 4 ). When a sufficient length of new filament was formed it continued the sinusoidal curve of the seed perfectly (Fig. 3) and the junction of seed and new filament always occurred without detectable deviation of the axis (Figs 3 and 4).

When HI I flagella were used as seed or monomer their strong polarity (Lawn et al., I977) established that polymerization of the new filament was always initiated from the forkshaped end, as described by Asakura et al. (1966). With flagellin from flagella of morphotypes $\mathrm{E}$ and $\mathrm{F}$, which apparently have sheaths, the sheath-like appearance was present throughout the length of the new filament.

The observation of J. Pye (quoted by Asakura et al., 1966) that polymerization was not initiated by flagella attached to bacteria, was confirmed.

\section{Co-polymerization of flagellins from flagella of two different morphotypes}

The results reported in the previous section, i.e. that flagellin from flagella of one morphotype would polymerize on to seed of fragments of flagella of an entirely different morphotype, suggested that the assembly pattern of the subunits of all these flagellar filaments was similar. It was decided to use a more stringent test for this hypothesis by attempting to assemble flagellin from flagella of two different morphotypes into one flagellar filament (Asakura et al., 1966). The polymerization experiments were performed as before except that instead of using a pure flagellin from one $\mathrm{H}$ serotype, the same volume of a mixture of two different flagellins was used. In different tubes the ratios of the volume of one flagellin solution to that of the other were I 25:I, 25:I, 5: I, I : I, I:5, I: 25, and I: I25. Three types of polymerization mixture were used, in each case with seed of $\mathrm{H}_{17}$ and with heat-dissociated flagellin from $\mathrm{H}_{5}$ as two of the components. The third component was heat-depoly- 
merized $\mathrm{H}_{7}, \mathrm{H}_{9}$ or $\mathrm{H}_{4} 6$ flagellin. As the amount of flagellin in each preparation varied and was not known, the ratio of the volumes of the flagellin solutions did not necessarily define the ratio of the numbers of molecules of each flagellin present per unit volume of the final polymerization mixture.

When the ratio of the volume of $\mathrm{H}_{5}$ flagellin to the volume of $\mathrm{H}_{7}, \mathrm{H}_{9}$ or $\mathrm{H}_{4} 6$ flagellin was 5 : $\mathrm{I}$ or more, long lengths of $\mathrm{H}_{5}$ filament were polymerized on to the $\mathrm{H}_{7} 7$ seed fragments and the presence of the other types of flagellin in the polymer could not be convincingly demonstrated, but isolated molecules might well have been undetectable. When the ratio was I : I 25, short segments of $\mathrm{H}_{7}$ or $\mathrm{H}_{4} 6$ filament were found on some of the seed fragments but there was no detectable polymerization of $\mathrm{H}_{5}$ filaments. No polymerization of any kind was detected at this ratio when the second flagellin was from $\mathrm{H} 9$ flagella. With I : I mixtures of $\mathrm{H}_{5}$ and either $\mathrm{H}_{7}$ or $\mathrm{H}_{4} 6$ flagellin solutions, short segments of filaments were polymerized on some seed fragments but their surface structure was not completely like that of either of the parent flagella (Fig. 5). With a $\mathrm{I}$ : $\mathrm{I}$ mixture of $\mathrm{H}_{5}$ and $\mathrm{H}_{9}$ flagellin solutions there was no detectable polymerization. The interpretation of these results must be influenced by the fact that when these same $\mathrm{H}_{5}, \mathrm{H}_{7}, \mathrm{H}_{9}$ and $\mathrm{H}_{4} 6$ flagellin solutions were mixed individually with suitable seed, $\mathrm{H}_{5}$ polymerized excellently, $\mathrm{H}_{7}$ and $\mathrm{H}_{4} 6$ poorly and in only short segments, and $\mathrm{H} 9$ showed no convincing evidence of polymerization.

\section{DISCUSSION}

Two principal hypotheses can be advanced to explain the differences in surface structure between $E$. coli flagella of different morphotypes. One assumes that some of the differences arise from the presence in some morphotypes of two layers of protein molecules, one forming the core and the other a separate sheath (Lowy \& Hanson, I965; Schmitt et al,, I974), whereas the other supposes that all the flagella are constructed from only one layer of flagellin molecules and that the different surface structure results from differences in the arrangement of the outer parts of these molecules. Three types of evidence in favour of the second hypothesis are provided by results reported here. The first is that only one major polypeptide band was present when acid- or heat-soluble protein from purified flagella of I3 different serotypes was examined by SDS-polyacrylamide gel electrophoresis. Five of these serotypes were from morphotypes $\mathrm{E}$ and $\mathrm{F}$ which are apparently sheathed (Lawn et al., I977), or six if H9 is accepted as having a sheath-like surface. Thus, unless in all six cases the molecular weight of the sheath protein is indistinguishable from the accompanying core protein using this technique, there is no separate sheath protein. The sheath protein might be insoluble in acid conditions or after heating, but samples of the same solutions of flagellin that polymerized to form normal filaments, apparently sheathed, gave single bands in SDSpolyacrylamide gel electrophoresis. Schmitt et al. (1974) also found a single flagellin band using apparently sheathed flagella from Pseudomonas rhodos.

The second type of evidence arises from a comparison between the molecular weight of a flagellin and the diameter of the corresponding flagella (Fig. 2). If a flagellum has a separate sheath layer, then its diameter will be the sum of the thicknesses of the core and the sheath layer. Variation of the molecular weight of one of these proteins would not be expected to give a simple relationship between diameter and molecular weight. However, Fig. 2 shows that the relationship between $d^{2}$ and molecular weight is approximately linear, and that the flagellins of apparently sheathed flagella are not distributed differently from those of unsheathed flagella.

The third type of evidence arises from the observation that throughout this investigation not one of the so-called sheathed flagella was ever seen as a sheathless core of constant diameter in even part of a filament. This was true of sonicated flagella, of newly polymerized flagella and of flagella in various stages of dissociation as a result of acid conditions.

The results of polyacrylamide gel electrophoresis of flagellins and those summarized in 
Fig. 2 do not exclude the possibility that the same protein, assembled in different ways, forms both sheath and core (Schmitt et al., I974), but an analysis of the results of the polymerization experiments provides further evidence against the presence of a separate sheath. In this case the core of the flagellum would be a complete layer of protein molecules and comparable to the entire filament of a non-sheathed flagellum. Measurements of the diameter of this core show that it has approximately the same diameter as that of the entire filament of the flagella of morphotype A, i.e. those of serotypes $\mathrm{H}_{4}$ and $\mathrm{Hr}_{7}$. However, the molecular weight of the flagellins from apparently sheathed flagella is in every case considerably greater than the molecular weight of $\mathrm{H}_{4}$ and $\mathrm{H}_{1} 7$ flagellins. If two molecules of very different molecular weight are to be assembled into a rod of the same diameter then there will either be considerably more fluid-filled space within the rod formed from the smaller molecules (for example, $\mathrm{H}_{4}$ or $\mathrm{H}_{1} 7$ flagella), for which there is no evidence, or the number of molecules per unit length, and therefore the assembly pattern of the molecules, must differ in the two cases. If it can be shown, on the contrary, that the assembly pattern is the same in both instances, then a consideration of the molecular weight relationships rules out the presence of a separate sheath.

The ability of one type of flagellar filament to polymerize on to seed consisting of fragments of another without detectable deviation of the axial direction suggests that the assembly pattern of the two types is nearly, if not absolutely, identical and it is shown here that thin, unsheathed flagella and thicker flagella that are apparently sheathed can be crosspolymerized in this way. The ability of flagellin from apparently sheathed flagella and flagellin from unsheathed flagella to co-polymerize, forming a rod with the form of a flagellum which continues the axis of the seed but has a surface structure indicating a mixture of the two types of flagellin, is even stronger evidence. It is difficult to believe that molecules with bonding regions adapted to the formation of one helical lattice could be inserted into spaces in another helical lattice without disrupting assembly, unless the two lattices were nearly identical. There is evidence that defective flagellin molecules disrupt assembly (see below).

If the evidence from cross-polymerization and co-polymerization is accepted as proving that the flagellin subunits are assembled into a nearly identical lattice in both apparently sheathed and in unsheathed flagella of $E$. coli then, as discussed above, a separate sheath cannot be present. In this case the linear relationship between $d^{2}$ and molecular weight is easily explained for, in an identical lattice, a large molecule must have a greater radial extent than a small molecule. The variation from an exact linear relationship in Fig. 2 can be attributed to the variation in degree of space-filling by flagellin in different types of flagella which is demonstrated by the differing extent of penetration of the negative-contrast agent, and also to inaccuracies of measurement.

A model of the structural gene for the flagellin of E. coli can be constructed to explain these observations. This hypothesis assumes that the gene can be divided into two parts, which may be termed the constant and variable regions. One end of the polypeptide, the code for which occupies the constant part of the gene, folds to form the part of the flagellin molecule which is innermost in the assembled flagellar filament, and which carries the specific bonding regions required to assemble a rigid flagellum possessing a spiral conformation. Mutations in this region of the gene are contra-selected as they abolish motility either because the flagellin cannot be assembled or because the resulting filament is unsatisfactory as a propulsive organ, for example straight or flexible. Martinez et al. (I968) have presented evidence that a single amino-acid substitution can produce a straight flagellum; this substitution would correspond to one in the constant part of the flagellin gene of $E$. coli. The part of the polypeptide whose code occupies the variable region of the gene lies on the outside of the flagellum and plays no part in its assembly so that massive insertions or deletions are permissible. This part is responsible for the surface properties of the flagellum including the exposed antigenic determinants, and mutations may frequently be advantageous because they allow the bacterium to escape the effect of the immobilizing antibody produced by the 
host organism. Such mutations would be responsible for creating the different H serotypes of E. coli.

The surface structures seen in different morphotypes must, then, be the result of different arrangements of the outer portions of the flagellum molecules, but the nature of the associations between neighbouring molecules is not immediately apparent. For example, the surface pattern of $\mathrm{H}_{5}$ flagella is relatively simple, but a calculation of the surface area occupied by a space-filling protein molecule of molecular weight 46000 in a filament $2 \mathrm{I} \mathrm{nm}$ in diameter gives an area three or four times less than that occupied by each apparent subunit. There is no evidence from the degree of penetration of the contrast agent to suggest that the molecules are widely separated from each other in $\mathrm{H}_{5}$ flagella and so it must be assumed that an apparent subunit is composed of several neighbouring polypeptide chains. Of course the subunit-like pattern might arise from the superimposition of front and back images of a flagellar filament possessing helical surface grooves which are not further subdivided.

An observation that requires comment is that some flagellin preparations only polymerized to form short lengths of filament, whose length was relatively constant even when different types or amounts of seed were used. The most likely explanation is that a proportion of flagellin molecules were denatured, and when by chance one, or a group, of these defective molecules was attached at the growing end of the filament further addition of molecules was prevented. This finding suggests a similar explanation for the observation that an $\mathrm{H}_{9}$ flagellin preparation gave no detectable polymerization but inhibited the polymerization of $\mathrm{H}_{5}$ flagellin; the majority of the $\mathrm{H}_{9}$ flagellin molecules might have been defective. Many other explanations for the phenomenon are, however, possible, and the polymerization of $\mathrm{H} 9$ monomer on $\mathrm{H}_{9}$ seed was not investigated.

The author is grateful to the Collaborative Centre for Reference and Research on Escherichia coli and Drs F. and I. Ørskov for the generous gift of bacterial strains and to Ivor Osborne for excellent technical assistance.

\section{REFERENCES}

AsakURA, S. \& EGUCHI, G. (1964). Reconstitution of bacterial flagella in vitro. Journal of Molecular Biology I0, 42-56.

ASAKURA, S., EGUCHI, G. \& IINo,T. (1966). Salmonella flagella: in vitro reconstruction and overall shapes of flagellar filaments. Journal of Molecular Biology 16, 302-316.

LAWN, A. M., Ørskov, I. \& ØRskov, F. (1977). Morphological distinction between different $\mathrm{H}$ serotypes of Escherichia coli. Journal of General Microbiology ror, I I I-I 19.

LowY, J. \& HANSON, J. (I965). Electron microscope studies of bacterial flagella. Journal of Molecular Biology I1, 293-313.

MaIzel, J. V. (197I). Polyacrylamide gel electrophoresis of viral proteins. In Methods in Virology, vol. 5, pp. 179-246. Edited by K. Maramorosch and H. Koprowski. New York: Academic Press.

MARTINez, R. J., ICHIKI, A. T., LUNDH, N. P. \& TronicK, S. R. (I968). A single amino acid substitution responsible for altered flagella morphology. Journal of Molecular Biology 34, 559564.

SCHMITt, R., RASKa, I. \& MAYer, F. (I974). Plain and complex flagella of Pseudomonas rhodos: analysis of fine structure and composition. Journal of Bacteriology 117, 844-857.

SMIth, R. W. \& KoffLeR, H. (I971). Bacterial flagella. In Advances in Microbial Physiology, vol. 6, pp. 219-339. Edited by A. H. Rose and J. F. Wilkinson. London: Academic Press. 